\title{
The Design of Simulator for Inter-Harmonic Detection with ESPRIT
}

\author{
${ }^{1}$ Nedim Aktan Yalcin and ${ }^{* 1}$ Fahri Vatansever \\ ${ }^{*}$ Faculty of Engineering, Electrical-Electronics Engineering Dept., Bursa Uludağ University, Turkey
}

\begin{abstract}
Inter-harmonics which exist in non-integer multiples of main harmonics cause power quality problems. In this study, in order to overcome power quality problems, ESPRIT algorithm which is one of the methods used for inter-harmonic detection which couldn't be accomplished by conventional spectrum analysis methods such as Fast Fourier Transform was realized in MATLAB environment. Software which was prepared in MATLAB environment has properties which are creating input signals, importing external signals, plotting frequency spectrum which is computed from obtained values with ESPRIT and presenting relative error rate of real signal values and ESPRIT algorithm values.
\end{abstract}

Key words: Inter-harmonics, ESPRIT, Simulator.

\section{Introduction}

In power systems, harmonic problem has existed since the emergence of alternating current (AC) systems and many studies have been proposed for definition and determination of this problem since beginning of $20^{\text {th }}$ century. Due to the fact that linear systems were generally used in the early stages of power systems, harmonics which were encountered in power systems were multiples of the main harmonics. Revealing these types of harmonics can easily be performed with commonly known techniques such as Fourier Transform. For this reason, in the early period of power electronics, harmonic analysis was not a conspicuous issue. But, at the end of $20^{\text {th }}$ century, inter-harmonics became an important issue depending on the development of semiconductor technology and its technological applications which include non-linear circuits such as converters, inverters, cycloconverters, rectifiers, etc. Inter-harmonics which are produced by non-linear circuits couldn't be determined with conventional harmonic analysis methods. Therefore, many techniques have been developed on inter-harmonic analysis [1-5].

Inter-harmonic determination methods could be separated in three classes: parametric methods, non-parametric methods and hybrid methods (Figure 1) [6]. Parametric methods define signal parameters (amplitude, phase and frequency) with mathematical equation and solve this equation stochastically or deterministically (such as MUSIC, ADALINE, Kalman Filter, Estimation of Signal Parameters via Rotational Invariance Techniques (ESPRIT), Prony etc.) [1,2,7-10]. On the other hand, non-parametric methods are such algorithms that could decompose signal's attributes (amplitude, phase and frequency) without depending on mathematical equations (such as Discrete Fourier Transform, Hilbert-Huang Transform, Wavelet Transform etc.) [11-15]. Lastly, hybrid methods are combination of parametric and non-parametric methods for determining signal components. The most important feature of hybrid methods are that their

*Corresponding author: Address: Faculty of Engineering, Department of Electrical-Electronics Engineering Bursa Uludağ University, 16059, Bursa TURKEY. E-mail address: fahriv@uludag.edu.tr, Phone: +902242940905 
capability to blend advantages of parametric and non-parametric methods and creating more robust method. There are many hybrid methods which are created by combining parametric and non-parametric methods [16-19].

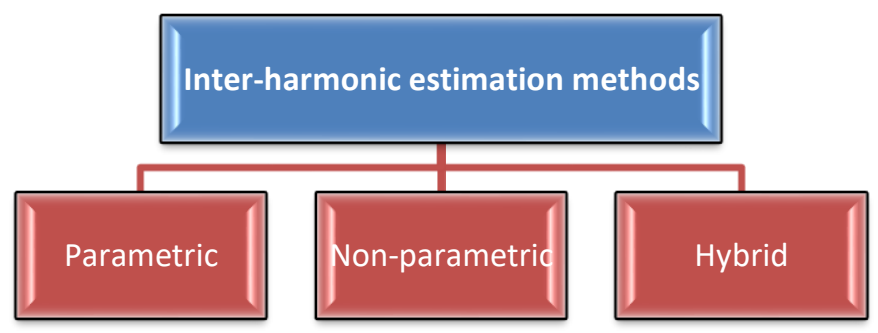

Figure 1. Classification of inter-harmonic estimation methods

In this study, ESPRIT method which belongs to parametric methods is investigated. Simulator program which is written in MATLAB [20] is designed for educational purposes. In this simulator, users can produce signals which are obtained from type IV energy conversion model [21], analyze produced signals and obtain related results graphically and numerically.

\section{Materials and Method}

In this section, chosen data model and ESPRIT method are explained. Data model is based on AC-DC-AC conversion system. ESPRIT algorithm is described with related data model. Details are explained in sub-sections.

\subsection{Data model}

Using realistic data are important for harmonic analysis. Because, randomly selected harmonic signals may be lack of signal processing issues such as picket-fence effect and spectral leakage. On the other hand, obtaining realistic data can be inconvenient and costly due to necessary for complex power system circuits. In order to overcome these difficulties, verified data models can be used for production of harmonic signals.

Although it is known that inter-harmonics are produced by non-linear loads, there are a few studies which focus on their creation mechanism [21-24]. According to these works, harmonic sources can be classified in four sub-concepts. They are AC-DC-AC conversion systems, AC-AC conversion systems, Time varying loads and continuously varying power production in system which are wind turbines, solar panels, etc.

In this work, type IV energy conversion system model are selected for signal production. This data model is verified with carried out measurements and appropriate for this work. Interharmonics which are produced by type IV systems are caused by ripples at DC link voltage of AC-DC-AC conversion system. In this model, it is assumed that, input frequency of this system is constant. 
Discussed system contains 3-phase rectifier, and single phase DC-AC inverter. Input frequency of rectifier is $f_{1}$. Frequencies of voltage across diodes are $6 f_{1}$ and output capacitor is affected with this frequency. If output capacitor has infinite value, there would be no voltage drop and so no inter-harmonic [23]. But in real systems, it is not possible to avoid capacitor discharge at output. Ripple at output has harmonics of integer multiple of input frequency $\left(6 f_{1}, 12 f_{1}, 18 f_{1}, \ldots\right)$.

Creation of inter-harmonics is carried out at DC-AC conversion step. Inverter modulates input signals with its system frequency is $f_{o}$. Therefore, inter-harmonics occur at these frequencies: $6 f_{1} \pm f_{0}, 12 f_{1} \pm f_{0}, 18 f_{1} \pm f_{0}, \ldots$ There is also an another inter-harmonic source, non-ideal character of inverter. Effect of non-ideality of AC-DC conversion systems are modeled in $[23,25,26]$. In investigated work, frequencies are grouped leading and lagging and expressed here as Equation 1 and Equation 2.

$$
\begin{aligned}
& f_{\text {leading }}^{k, n}=\left|6 k f_{1}+(6 n \pm 1) f_{0}\right| \\
& f_{\text {lagging }}^{k, n}=\left|6 k f_{1}-(6 n \pm 1) f_{0}\right|
\end{aligned}
$$

\subsection{ESPRIT}

In this section, ESPRIT algorithm is explained in two stages. Firstly, signal model is constructed then estimation principles are explained. All equations in this section based on these papers, [2, 27-28]

\subsubsection{Signal model}

In established signal model, it is considered that studied signal is narrowband signal. Equation 3 defines a generalized signal in complex form. If a generalized signal written in sinusoidal form as in Equation 4, it can also be converted notation of Equation 3

$$
\begin{gathered}
s(t)=\alpha e^{j \beta} e^{j \omega t}=\rho e^{j \omega t} \\
\alpha \cos (\omega t+\beta)=\frac{\alpha}{2} e^{j \beta} e^{j \omega t}+\frac{\alpha}{2} e^{-j \beta} e^{-j \omega t} \\
=\rho_{1} e^{j \omega t}+\rho_{2} e^{-j \omega t}
\end{gathered}
$$

In presence of phase shift in signal, Equation 5 can be written. This equation is approximately applied when considered narrowband signals.

$$
\begin{gathered}
s\left(t-t_{0}\right)=e^{-j \omega t_{0}} \rho e^{j \omega t} \\
=e^{-j \omega t_{0}} s(t)
\end{gathered}
$$

With generalization of above equations, multiple signal case can be established. In multiple signal case, it is assumed that all signals have uncorrelated amplitudes and different frequencies. Expectation of signals amplitudes is given in Equation 6. 


$$
E\left\{\rho_{i}, \rho_{j}\right\}=\left\{\begin{array}{c}
\sigma_{i}^{2}, i=j \\
0, i \neq j
\end{array}\right.
$$

Due to origin of ESPRIT algorithm depends on Antenna Theory, it will be convenient assuming incident signal is captured by $\mathrm{N}$ sensors which are apart from each other with distance $d$. If captured is $s(t)$ by first sensor, and $i$. delay is represented by $\Delta_{i}$, Equation 7 and Equation 8 can be written.

$$
\begin{gathered}
\Delta_{i}=\frac{(i-1) d \sin \theta}{c} \\
x_{i}(t)=e^{-j \omega \Delta_{i_{0}}} S(t)=e^{-j \omega \frac{(i-1) d \sin \theta}{c}} S(t)
\end{gathered}
$$

After defining these equations, signal model can be constructed. Captured signals by sensors have noise, so this effect is considered when deriving signal model. In Equation 9, $\mathbf{a}(\theta)$ is called as steering vector.

$$
\begin{gathered}
x(t)=\left[\begin{array}{c}
x_{1}(t) \\
x_{2}(t) \\
\vdots \\
x_{N}(t)
\end{array}\right]=\left[\begin{array}{c}
1 \\
e^{-j \omega \frac{d \sin \theta}{c}} \\
\vdots \\
e^{-j \omega \frac{(N-1) d \sin \theta}{c}}
\end{array}\right] s(t) \\
=\mathbf{a}(\theta) s(t)
\end{gathered}
$$

If there are independent $I$ source of signal, signal model can be written as Equation 10. In this equation, $\mathbf{x}(t)$ is received signal $N \times 1, \mathbf{s}(t)$ is source signal vector $(I \times 1) \mathbf{n}(t)$ is noise vector $(N \times 1) \mathbf{A}$ is steering vector $(N \times I)$.

$$
\mathbf{x}(t)=\mathbf{A s}(t)+\mathbf{n}(t)
$$

\subsubsection{Algorithm}

Equation 10 can be written as Equation 11

$$
\begin{aligned}
\mathbf{y}(t)= & \mathbf{x}(t+1)=\mathbf{A} \mathbf{s}(t+1)+\mathbf{n}(t+1) \\
& =\mathbf{A} \boldsymbol{\phi} \mathbf{s}(t+1)+\mathbf{n}(t+1) \\
\boldsymbol{\phi} & =\operatorname{diag}\left(e^{j \omega_{1}}, e^{j \omega_{2}}, \ldots, e^{j \omega_{N}}\right)
\end{aligned}
$$

Auto correlation and cross correlation matrix is given Equation 12 and Equation 13

$$
\begin{gathered}
R_{x, x}=E\left\{\mathbf{X}(t) \mathbf{X}^{H}(t)\right\}=\mathbf{A} \mathbf{P} \mathbf{A}^{H}+\sigma^{2} \mathbf{I} \\
R_{x, y}=E\left\{\mathbf{X}(t) \mathbf{Y}^{H}(t)\right\}=\mathbf{A} \mathbf{P} \boldsymbol{\phi}^{H} \mathbf{A}^{H}+\sigma^{2} \mathbf{Z}
\end{gathered}
$$

In Equation (12) and Equation (13), $\sigma^{2}$ is white noise, $\mathbf{I}$ is identity matrix, $\mathbf{Z}$ is offset form of identity matrix by one element and $\mathbf{P}=E\left\{\mathbf{S}(t) \mathbf{S}^{H}(t)\right\}$. Finally, frequencies are estimated with calculating generalized eigenvalue of $\mathbf{C}_{\mathbf{x}, \mathbf{x}}=\mathbf{A} \mathbf{P} \mathbf{A}^{H}$ and $\mathbf{C}_{\mathbf{x}, \mathbf{y}}=\mathbf{A} \mathbf{P} \boldsymbol{\phi}^{H} \mathbf{A}^{H}$ 


\section{Simulations}

In this section, MATLAB simulation of educational tool is presented. Screenshot of simulator is seen in Figure 2. In realized simulator, users can load or create signals, analyze signals with ESPRIT algorithm and obtain numerical results which belong to signal attributes.

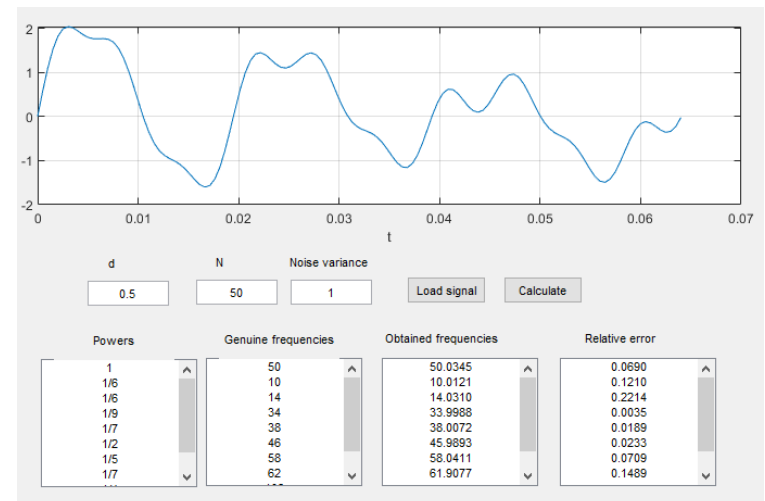

Figure 2. Designed simulator

In this section, Equation 1 and Equation 2 (derived from [21]) are used for frequency generation. 14 coefficients for each frequency subgroup are considered. Due to presence of four frequency subgroup, 56 frequencies are investigated. Then six harmonics are considered. Powers of harmonics are randomly selected. Input frequency of rectifier is $58 \mathrm{~Hz}$ and output frequency of DC-AC conversion unit is $50 \mathrm{~Hz}$. Signal model from Equation 10 is implemented. Variance of noise is selected as 1 . Obtained results are shown in Table 1. In this table, column which is named "genuine value" represents real value of output frequency of inverter. Column which is called "detected value" is calculated result by ESPRIT algorithm. Last column shows related error of obtained results.

Table 1. Detection of frequencies with ESPRIT algorithm

\begin{tabular}{lllll}
\hline $\begin{array}{l}\text { Frequency } \\
\text { number }\end{array}$ & Power & $\begin{array}{l}\text { Genuine } \\
\text { value }\end{array}$ & $\begin{array}{l}\text { Detected } \\
\text { value }\end{array}$ & $\begin{array}{l}\text { Relative } \\
\text { error }\end{array}$ \\
\hline 1 & 1 & 50 & 50.0345 & 0.0690 \\
2 & $1 / 6$ & 10 & 10.0121 & 0.1210 \\
3 & $1 / 6$ & 14 & 14.0310 & 0.2214 \\
4 & $1 / 9$ & 34 & 33.9988 & 0.0035 \\
5 & $1 / 7$ & 38 & 38.0072 & 0.0189 \\
6 & $1 / 2$ & 46 & 45.9893 & 0.0233 \\
7 & $1 / 5$ & 58 & 58.0411 & 0.0709 \\
8 & $1 / 7$ & 62 & 61.9077 & 0.1489 \\
9 & $1 / 4$ & 106 & $\sim$ & $\sim$ \\
10 & $1 / 3$ & 154 & $\sim$ & $\sim$ \\
\hline
\end{tabular}

In table 1, it is shown that low frequencies are detected more accurately than high frequencies 
which are shown in last 2 rows. It is due to narrow band modeling of this algorithm. Different and more accurate results can be obtained with changing $d$ parameter in Equation 8 . In this simulation, $d$ is selected as 0.5

\section{Conclusion}

In this study, harmonic analysis software was developed. In this software, users can analyse signals with ESPRIT algorithm. Due to parametric nature of implemented algorithm, not only integer harmonics but also inter-harmonics which are non-integer multiples of main harmonics can be calculated. Obtained results can be presented numerically and graphically. Realized software can also be used for educational purposes owing to its interactive interface. It can be used for understanding of harmonic analysis concept by students.

\section{References}

[1] Schmidt R. Multiple Emitter Location and Signal Parameter. IEEE Trans Antennas Prop 1986.

[2] Roy R, Kailath T. ESPRIT-estimation of signal parameters via rotational invariance techniques. IEEE Trans Acoust 1989;37:984-95. doi:10.1109/29.32276.

[3] Gu IY-H, Bollen MHJ. Estimating Interharmonics by Using Sliding-Window ESPRIT. IEEE Trans Power Deliv 2008;23:13-23. doi:10.1109/TPWRD.2007.911130.

[4] Jiefeng Xiong, Boling Wang, Shaoyong Zhang. Interharmonics analysis based on windowed interpolation and prony algorithm. 2010 2nd Int. Asia Conf. Informatics Control. Autom. Robot. (CAR 2010), IEEE; 2010, p. 430-3. doi:10.1109/CAR.2010.5456806.

[5] Huang $\mathrm{CH}$, Lee $\mathrm{CH}$, Shih KJ, Wang YJ. A robust technique for frequency estimation of distorted signals in power systems. IEEE Trans Instrum Meas 2010;59:2026-36. doi:10.1109/TIM.2009.2028776.

[6] Jain SK, Singh SN. Harmonics estimation in emerging power system: Key issues and challenges. Electr Power Syst Res 2011;81:1754-66. doi:10.1016/J.EPSR.2011.05.004.

[7] Chang GW, Cheng-I Chen, Quan-Wei Liang. A Two-Stage ADALINE for Harmonics and Interharmonics Measurement. IEEE Trans Ind Electron 2009;56:2220-8. doi:10.1109/TIE.2009.2017093.

[8] Dash PK, Pradhan AK, Panda G. Frequency estimation of distorted power system signals using extended complex Kalman filter. IEEE Trans Power Deliv 1999;14:761-6. doi:10.1109/61.772312.

[9] Kalman RE. A New Approach to Linear Filtering and Prediction Problems. J Basic Eng 1960;82:35-45. doi:10.1115/1.3662552.

[10] Prony GRB. Essai experimantal et analytique, etc. J L'Ecole Polytech 1795;1:24-76.

[11] Cooley JW, Tukey JW. An Algorithm for the Machine Calculation of Complex Fourier Series. Math Comput 1965;19:297. doi:10.2307/2003354.

[12] Grossmann A, Morlet J. Decomposition of Hardy Functions into Square Integrable Wavelets of Constant Shape. SIAM J Math Anal 1984;15:723-36. doi:10.1137/0515056.

[13] Huang NE, Shen Z, Long SR, Wu MC, Shih HH, Zheng Q, et al. The empirical mode decomposition and the Hilbert spectrum for nonlinear and non-stationary time series 
analysis. Proc R Soc A Math Phys Eng Sci 1998;454:903-95. doi:10.1098/rspa.1998.0193.

[14] Mallat SG. A theory for multiresolution signal decomposition: the wavelet representation. IEEE Trans Pattern Anal Mach Intell 1989;11:674-93. doi:10.1109/34.192463.

[15] Winograd S. On computing the Discrete Fourier Transform. Proc Natl Acad Sci 1976;73:1005-6. doi:10.1073/pnas.73.4.1005.

[16] Bettayeb M, Qidwai U. A hybrid least squares-GA-based algorithm for harmonic estimation. IEEE Trans Power Deliv 2003;18:377-82. doi:10.1109/TPWRD.2002.807458.

[17] Hostetter GH. Recursive Discrete Fourier Transformation. IEEE Trans Acoust 1980;28:184-90. doi:10.1109/TASSP.1980.1163389.

[18] Mishra S. A Hybrid Least Square-Fuzzy Bacterial Foraging Strategy for Harmonic Estimation. IEEE Trans Evol Comput 2005;9:61-73. doi:10.1109/TEVC.2004.840144.

[19] Tarasiuk T. Hybrid wavelet-Fourier spectrum analysis. IEEE Trans Power Deliv 2004;19:957-64. doi:10.1109/TPWRD.2004.824398.

[20] Mathworks.com. Matlab. The MathWorks 2019. http://mathworks.com.

[21] Yang K, Bollen MHJ. Interharmonic currents from a Type-IV wind energy conversion system. Electr Power Syst Res 2017. doi:10.1016/j.epsr.2016.09.010.

[22] Marz MB. Interharmonics: What They Are, Where They Come From and What They Do. Minnesota Power Syst Conf Pap 2016. doi:10.1017/CBO9781107415324.004.

[23] Testa A, Akram MF, Burch R, Carpinelli G, Chang G, Dinavahi V, et al. Interharmonics: Theory and modeling. IEEE Trans Power Deliv 2007. doi:10.1109/TPWRD.2007.905505.

[24] Sangwongwanich A, Yang Y, Sera D, Soltani H, Blaabjerg F. Analysis and Modeling of Interharmonics from Grid-Connected Photovoltaic Systems. IEEE Trans Power Electron 2018. doi:10.1109/TPEL.2017.2778025.

[25] De Rosa F, Langella R, Sollazzo A, Testa A. On the interharmonic components generated by adjustable speed drives. Proc. Int. Conf. Harmon. Qual. Power, ICHQP, 2002. doi:10.1109/ICHQP.2002.1221430.

[26] Yacamini R. Power system harmonics. Part 4 Interharmonics. Power Eng J 1996. doi:10.1049/pe:19960411.

[27] Sun X, Zhe C, Wei T. Harmonic frequency estimation algorithm based on ESPRIT and MSWF in power system. Open Electr Electron Eng J 2015;9:518-23. doi:10.2174/1874129001509010518.

[28] GIRD Systems I. An Introduction to MUSIC and ESPRIT. GIRD Syst Inc n.d. http://www.girdsystems.com/pdf/GIRD_Systems_Intro_to_MUSIC_ESPRIT.pdf (accessed October 14, 2019). 